\title{
Rationale and design of the PLACID study: a randomised trial comparing the efficacy and safety of inhaled loxapine versus IM aripiprazole in acutely agitated patients with schizophrenia or bipolar disorder
}

\author{
L. San ${ }^{1 *}$, G. Estrada ${ }^{2}$, N. Oudovenko ${ }^{2}$ and E. Vieta ${ }^{3}$
}

\begin{abstract}
Background: The management of acute agitation manifesting in patients with schizophrenia or bipolar disorder requires swift pharmacological intervention to provide rapid symptomatic relief and prevent escalation to aggression and violence. Antipsychotic medications are widely used in this setting and the availability of an inhaled formulation with deep lung absorption of the antipsychotic loxapine has the potential to deliver a faster onset of therapeutic effect than the available intramuscular formulations of antipsychotics.

Methods: The efficacy of inhaled loxapine and the alternative antipsychotic aripiprazole delivered via intramuscular (IM) injection will be compared in the Phase IIIb PLACID study. Adults (18-65 years) with a confirmed diagnosis of schizophrenia or bipolar I disorder presenting with acute agitation will be randomly assigned to open-label treatment in a 1:1 ratio. Clinical evaluation will be conducted by raters blinded to treatment assignment. The primary efficacy endpoint is time to response (defined as a Clinical Global Impression of Improvement [CGI-I] score of 1 [very much improved] or 2 [much improved]).

Secondary endpoints will include the percentage of responders at different time points after dosing; the proportion of patients who receive 1 or 2 doses of study drug; time to second dose; time to rescue medication; satisfaction with study drug (evaluated using Item 14 of the Treatment Satisfaction Questionnaire for Medication); and safety and tolerability. Approximately 360 patients will be recruited with an interim analysis conducted once 180 patients have completed the study to decide whether to stop for futility or continue with or without an increase in the sample size up to additional 288 patients.
\end{abstract}

Discussion: The PLACID trial will assess the efficacy and safety of inhaled loxapine with deep lung absorption compared with the IM antipsychotic, aripiprazole, in acutely agitated patients with schizophrenia or bipolar disorder. In the event that the median time to response of inhaled loxapine is significantly shorter than that of the intramuscular aripiprazole, the PLACID study has the potential to support the inhaled antipsychotic therapy as the standard of care in this setting.

Trial registration: The study protocol was registered with the European Clinical Trials Database on the 31 October 2014 (EudraCT number 2014-000456-29).

Keywords: Acute agitation, Schizophrenia, Bipolar disorder, Antipsychotic, Inhaled-loxapine, IM-aripiprazole, Rapid onset

\footnotetext{
* Correspondence: Isan@pssjd.org

${ }^{1}$ Mental Health Department, Parc Sanitari Sant Joan de Deu, CIBERSAM,

Carrer Camí Vell de la Colonia, 25 Sant Boi de Llobregat Barcelona, Spain

Full list of author information is available at the end of the article
} 


\section{Background}

Schizophrenia and bipolar disorder are estimated to affect 21 million and 60 million individuals worldwide, respectively [1]. Agitation, defined as a state of cognitive and motor hyperactivity characterized by excessive or inappropriate motor or verbal activity with an emotional arousal element, is common among patients with schizophrenia or bipolar disorder [2-4]. For these patients, the manifestation of acute agitation represents a serious, disruptive and morbid complication that can escalate rapidly and unpredictably from distress to loss of control and physical aggression and violence towards themselves, other patients and their carers [4-6].

Acute agitation is frequently encountered in both medical and psychiatric emergency settings and should be managed initially with verbal de-escalation and environmental management $[7,8]$. Continued escalation of agitation may ultimately require physical restraint and pharmacological treatment [7, 8]. However, such coercive management measures may damage the therapeutic relationship between patients and their carer's and evoke feelings of panic, fear, powerlessness, anger, frustration, and injustice [2]. The consequence of this negative association of feelings may destroy trust and lead to patient reluctance to seek psychiatric care [3]. Early intervention for acute agitation with a medication delivered via a non-traumatic route has the potential to avoid the negative feelings evoked by a traumatic therapeutic encounter and reinforce the partnership between patient and their carer $[2,3]$.

The costs associated with agitation, especially agitation that escalates to aggression and violence, among patients with psychiatric disorders may also be considerable although precise estimates of the overall burden are lacking. When inadequately managed, agitation among psychiatric patients can result in an increase in the number and duration of inpatient hospital stays, a reduced likelihood of discharge to community and use of coercive measures and/or escalation to patient violence, presenting a substantial economic burden to the healthcare system [8, 9]. In Spain, the annual cost of psychiatric mechanical restraint has been estimated at $€ 27$ million [9]. A separate study conducted in the UK, estimated that the annual cost per psychiatric ward for containment was $€ 267,069$ in 2014 [10].

Agitation among patients with psychiatric disorders requires early recognition and swift intervention with treatments that can achieve rapid symptom relief and avoid the progression of agitation to aggression and violence [7]. The acute treatment of agitation associated with schizophrenia or bipolar disorder includes pharmacological tranquilization with oral antipsychotic agents given alone or in combination with benzodiazepines [11-13]. However, while a preferred route of delivery in terms of maintaining a non-traumatic experience for the patient, orally dosed agents may not be optimal in terms of delivering a sufficiently rapid onset of therapeutic effect. Intramuscular (IM) formulations have been shown to provide relatively faster symptomatic relief compared with oral formulation of antipsychotics but have the disadvantage of a potentially traumatic experience for the patient $[2,14]$.

Inhaled loxapine with deep lung absorption is the first anti-agitation medication available to provide rapid control of agitation combined with a noncoercive noninvasive route of administration. It is administered by inhalation using the Staccato delivery system. The efficacy of inhaled loxapine for the management of agitation among patients with schizophrenia or bipolar I disorder has been confirmed in placebo-controlled, Phase III trials [15-17] and is supported by indirect comparisons with other antipsychotics [18]. However, head-to-head comparisons with other antipsychotics, including those delivered via IM injection, are lacking. Head-to-head trials, often neglected by regulatory authorities and industry sponsors, are important for clinical practice [19]. The PLACID study will compare inhaled loxapine and IM aripiprazole (considered a standard of care in this setting; [20]) for the prompt stabilization of acutely agitated patients with schizophrenia or bipolar disorder.

\section{Methods}

The PLACID study will evaluate the efficacy, defined as time to response (Clinical Global Impression of Improvement [CGI-I] or 1 or 2) of inhaled loxapine as compared with aripiprazole administered via IM injection in acutely agitated patients with schizophrenia or bipolar disorder.

Inhaled loxapine is available in $10 \mathrm{mg}$ dose, being the delivered dose 9,1 mg, a maximum of 2 doses can be administered during $24 \mathrm{~h}$ (24). For IM aripiprazole the standard dose is $9,75 \mathrm{mg}$ with a possibility of two additional doses during $24 \mathrm{~h}$ (14). In this study a maximum of 2 doses of both study drugs are allowed [14, 21].

The study will be conducted at up to 30 centres across Europe including centres in the Czech Republic, Germany, Russia and Spain and will include male and female patients aged between 18 and 65 years with a confirmed diagnosis of schizophrenia or bipolar I disorder according to Diagnostic and Statistical Manual of Mental Disorders (DSM-5) criteria.

\section{Design}

The PLACID study is a Phase IIIb, open-label, rater blind, randomised, active control, prospective, parallel group, multicentre clinical trial (Fig. 1, Table 1). The study will be conducted in two phases. The first phase will enrol approximately 180 patients who will be 

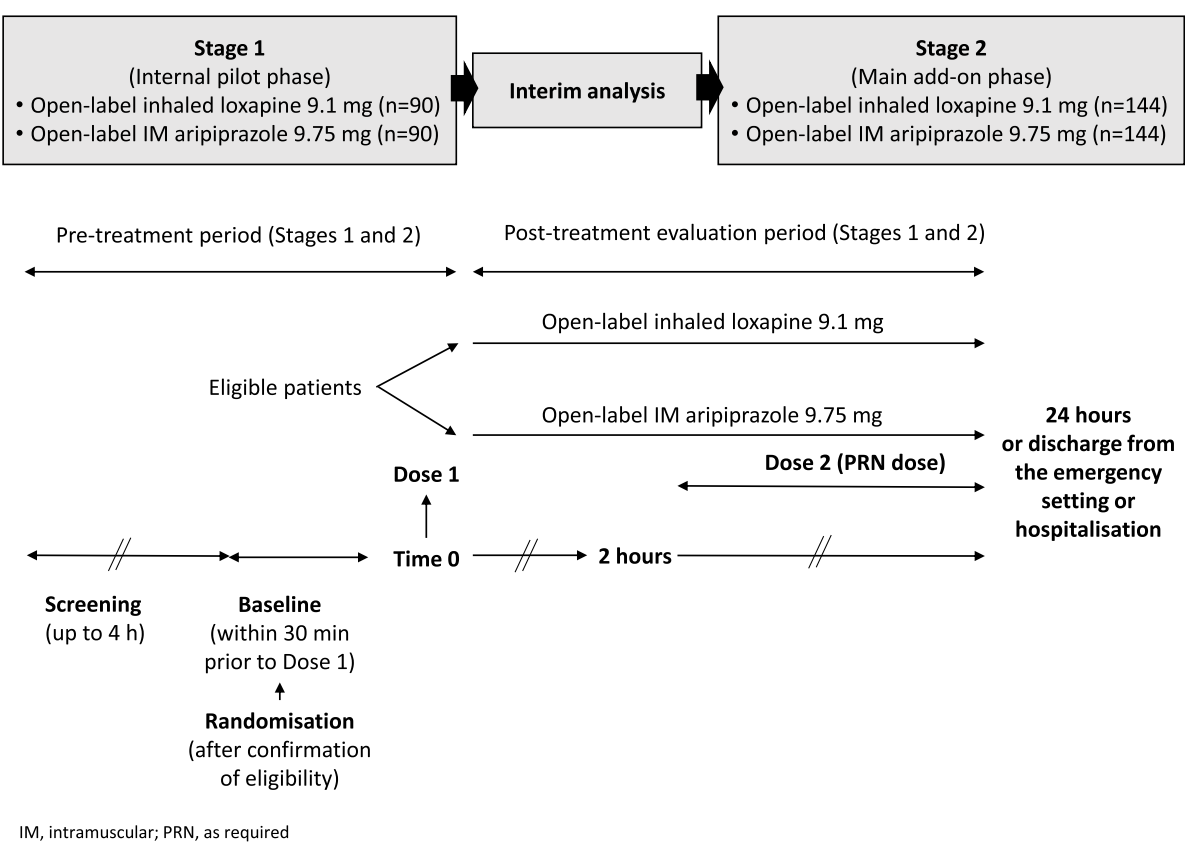

Fig. 1 Study design

randomly assigned (in a 1:1 ratio) to open-label treatment. Clinical evaluation will be carried out by personnel blinded to treatment assignment. Patients will be instructed not to tell the blinded assessor which treatment they received.

An interim analysis will be performed on the phase 1 data to determine whether to continue into phase 2 or to stop the trial due to futility or unfeasibility. The final sample size of the PLACID study will be re-calculated based on the interim analysis results. The two-phase design with interim analysis is justified due to the limited availability of CGI-I data during the first 2 h' postadministration both for inhaled loxapine and IM aripiprazole, and therefore making difficult the sample size estimation. Data demonstrating meaningful improvements from baseline on the CGI-I scale at 120 mins post dose are available [15, 16, 20, 22] for both treatments, but data demonstrating the time course of these improvements during the first two hours of treatment, when rapid and effective action is required to minimise the risks associated with acute agitation, are lacking.

\section{Patients}

The study population will consist of patients meeting DSM-5 criteria for schizophrenia or bipolar I disorder experiencing an acute episode of agitation, both hospitalized and attending to a psychiatric or general emergency room.

To be eligible to participate patients must be clinically agitated at baseline with a value $\geq 4$ for the Clinical Global Impression of Severity (CGI-S; [23]) scale and otherwise in good health. Patients will be specifically excluded from participation if their agitation is judged to be caused primarily by acute alcohol or drug intoxication/withdrawal. Those patients judged to be at serious risk for suicide will also be excluded. Patients treated with benzodiazepines or other hypnotics or oral or short-acting IM antipsychotics within $1 \mathrm{~h}$ prior to study drug administration will be excluded although those patients may be subsequently reassessed for inclusion. Other exclusion criteria will include pregnancy or breastfeeding, a history of or current significant hepatic, renal, gastroenterologic, respiratory, cardiovascular, endocrinologic, neurologic or haematologic disease as well as acute or active airways disease.

All patients will be required to provide written informed consent prior to undergoing any study procedure. Patients considered by appropriately qualified staff to be of impaired capacity to provide informed consent will not be eligible for participation. In Spain a proxy or deferred informed consent is possible. If patients have recovered the capacity, they will be asked to provide the informed consent; otherwise the data will not be used in the analysis.

\section{Study treatments}

Baseline evaluations will be performed as close as possible to, and within $30 \mathrm{~min}$ before, administration of initial study medication. Patients will be randomly assigned to receive either inhaled loxapine $10 \mathrm{mg}$ (delivered dose $9.1 \mathrm{mg}$ ) using the hand-held, portable, Staccato delivery system or IM aripiprazole $9.75 \mathrm{mg}$ per 


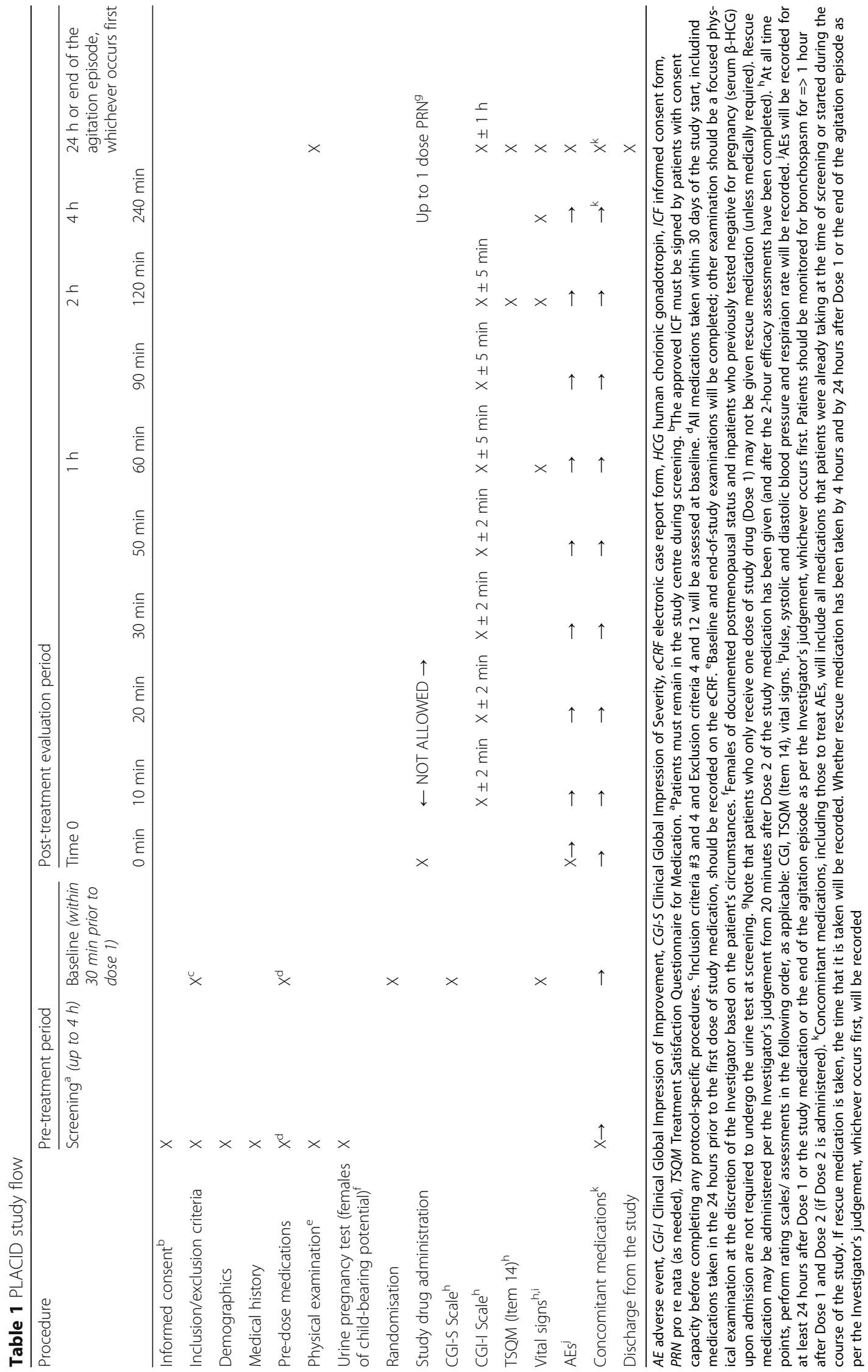


$1.3 \mathrm{~mL}$ dose. Patients will receive a maximum of 2 doses of either study drug with the second dose given if required at least $2 \mathrm{~h}$ after the first dose.

Randomisation will be achieved using an interactive voice response system and will be stratified according to the patient's disease type (schizophrenia or bipolar I disorder).

The planned duration of participation in the study will be up to approximately $30 \mathrm{~h}$ consisting of a screening and baseline phase of up to $4.5 \mathrm{~h}$ and a post-treatment period which will commence with the administration of the first dose of study drug and will continue for at least $4 \mathrm{~h}$ and up to a maximum of $24 \mathrm{~h}$.

\section{Outcomes and endpoints}

The primary efficacy endpoint is time to response measured during the first $2 \mathrm{~h}$ after the first dose. Response is defined as a CGI-I score of 1 (very much improved) or 2 (much improved). Secondary endpoints will include: the proportion of patients achieving response at 10, 20, 30, 50, 60, 90 and 120 min after the first dose of study drug; the total number of patients who receive 1 or 2 doses of study drug without rescue medication by $4 \mathrm{~h}$ and $24 \mathrm{~h}$ after the first dose of study drug; time to rescue medication; time to the second dose of study drug; and satisfaction with study drug (evaluated using Item 14 of the Treatment Satisfaction Questionnaire for Medication [TSQM]; [24]). Safety and tolerability will also be assessed through the reporting of adverse events, physical examination, recording of vital signs.

\section{Statistical analysis}

The primary endpoint "time to response" is defined as the first time point in minutes after Dose 1 at which CGI-I score of 1 or 2 is achieved during the $120 \mathrm{~min}$ after the first dose. The comparison of time to response between the two treatment groups will be performed using a Wilcoxon rank-sum test with a two-sided significance level of 0.05 . Patients without response within $2 \mathrm{~h}$ will be considered as non-responders and the time to response' as $4 \mathrm{~h}$ ( $2 \mathrm{~h}$ more than the maximum follow-up for this variable). The median difference $(95 \% \mathrm{CI})$ will be estimated by means of the Hodges-Lehmann approach based on the Wilcoxon rank-sum (Mann-Whitney) distribution. Kaplan-Meier curves of time to response will also be presented by treatment group. Additionally, the Mantel-Haenszel log-rank test will also be conducted for sensitivity purposes.

Secondary analyses will be exploratory without formal sample size estimation. Safety and tolerability data will be presented descriptively.

A total sample size of 180 in each group will have approximately $90 \%$ power to demonstrate superiority of inhaled loxapine versus IM aripiprazole for the primary efficacy endpoint at a one-sided significance level of 0.025 . If the interim analysis suggests that more than $30 \%$ additional patients are required at 0.025 one-tailed alpha level and approximately $90 \%$ power, it will be considered if it is feasible to include $>30 \%$ additional patients. Since there will be neither stopping for overwhelming efficacy, nor is there a situation where the sample size will decrease based on the interim analysis results, the alpha level will be preserved.

\section{Discussion}

Acute agitation in patients with psychiatric disorders including schizophrenia and bipolar disorder is a serious complication with the potential to escalate rapidly to aggression and physical violence $[3,5,6]$. Early recognition of the onset of acute agitation and intervention to rapidly calm the agitated patient without over-sedation are the main goals of the pharmacological management of such patients [3]. Antipsychotic medications are widely used in this setting and IM delivery provides a more rapid onset of effect compared with oral formulations. However, IM delivery of medication in this setting may not be optimal for patients already in an agitated state and may interfere with the therapeutic relationship between the patient and their carers [2]. Inhaled formulations have the potential to provide a more rapid onset of effect than IM injection. The medication is delivered through the lung with rapid transition to the systemic circulation via a non-coercive, non-invasive route of administration [3, 25]. Clinical evaluation of the inhaled antipsychotic loxapine has demonstrated an onset of therapeutic effect as early as 10 min after dosing, an effect that is maintained through $24 \mathrm{~h}$ among patients with acute agitation and schizophrenia or bipolar disorder $[15,16,21,26]$.

The PLACID trial will assess the efficacy and safety of inhaled loxapine compared with the IM antipsychotic, aripiprazole, in acutely agitated patients with schizophrenia or bipolar disorder. The PLACID trial is planned as a pragmatic trial close to the real world setting.

The CGI scale, which was developed as a brief, standalone assessment of the patient's global functioning prior to and after initiating a study medication, will be used to assess the primary endpoint. This is a simple instrument widely used in a clinical practice and can be completed in less than a minute by an experienced rater [27] Even if not validated for agitation assessment, it was shown that there is a very good correlation between the CGI and PANSS-EC [28]. All raters will be trained in the proper CGI use to assess the agitation grade.

Both inhaled loxapine and IM aripiprazole are currently available in Europe for the treatment of acute agitation in patients with schizophrenia or bipolar disorder. 
REMS (Risk Evaluation and Mitigation Strategy) is necessary with inhaled loxapine to mitigate the risk of bronchospasm. For this reason, only the centers with immediate access on site to supplies and personnel trained to manage acute bronchospasm will participate in this study. REMS is the strategy to manage a known or potential serious risk associated with a drug or biological product required by the US FDA (Food and Drug Administration).

The pharmacokinetic profiles (PK) of both drugs differ significantly and this is explained by the differences in the administration route. The peak plasma concentration of IM aripiprazole is achieved at $1 \mathrm{~h}$, while for inhaled loxapine it is $2-3 \mathrm{~min}$. Such a short time is explained by the fact that inhaled loxapine is directly absorbed into the blood stream with a PK profile similar to IV formulations. This study will prove if these differences in PK parameters correspond to clinical parameters, namely shorter time to response of inhaled loxapine.

Patients will be randomly assigned to treatment to avoid bias in terms of treatment allocation. The delivered treatment is necessarily open-label due to the different methods of administration. To avoid the issue of rater bias, drug administration and clinical evaluation will be carried out by different personnel, with the clinical rater blinded to treatment allocation and the randomization will be stratified by diagnosis to balance the groups. Patients will be instructed not to tell the treatment they receive.

\section{Conclusion}

In conclusion, the prompt stabilisation of patients with schizophrenia or bipolar disorder presenting with acute agitation has the potential to facilitate a patient-centered management approach by maintaining the patientphysician relationship and minimising the need for physical or mechanical restraint or sedation [2, 3]. The PLACID study will allow, for the first time, the direct comparison of the timing of the onset of therapeutic effect of an IM antipsychotic, aripiprazole, and an inhaled antipsychotic, loxapine, for the treatment of acute agitation in patients with schizophrenia or bipolar disorder. The results of this trial have the potential to change future treatment guidelines and have a powerful impact on clinical practice.

\section{Additional file}

Additional file 1: Ethics committees that approved the study: The contains the names and other relevant information of all the ethics committee that have approved the study. (XLS $59 \mathrm{~kb}$ )

\section{Abbreviations}

CGI-I: Clinical global impression of improvement; CGI-S: Clinical global impression of severity; DSM: Diagnostic and statistical manual of mental disorders; IM: Intramuscular; TSQM: Treatment satisfaction questionnaire for medication; WHO: World Health Organization

\section{Acknowledgements}

We would like to thank Tracey Lonergan and Alberto Moldón from Adelphi Spain for their help with the manuscript writing.

\section{Funding}

The PLACID study was funded by Ferrer Internacional, S.A.

\section{Availability of data and materials}

Not applicable.

\section{Authors' contributions}

LS: Contributed to the design, training, and study conduct, as well as the inception, preparation and approval of this manuscript. GE: Contributed to the study conduct, as well as the inception, preparation and approval of this manuscript. NO: Contributed to the study conduct, design, as well as preparation and approval of this paper. EV: Contributed to the design, training, and study conduct, as well as the inception, preparation and approval of this manuscript. All authors read and approved the final manuscript.

\section{Competing interests}

EV: Dr. Vieta has received grants and served as consultant, advisor or CME speaker for the following entities: AB-Biotics, Actavis, Allergan, AstraZeneca, Bristol-Myers Squibb, Ferrer, Forest Research Institute, Gedeon Richter, Glaxo-Smith-Kline, Janssen, Lundbeck, Otsuka, Pfizer, Roche, Sanofi-Aventis, Servier, Shire, Sunovion, Takeda, Telefónica, the Brain and Behaviour Foundation, the Spanish Ministry of Science and Innovation (CIBERSAM), the Seventh European Framework Programme (ENBREC), and the Stanley Medical Research Institute. GE: Dr. Estrada is Director Clinical Development at Ferrer. NO: N. Oudovenko is Clinical Research Manager at Ferrer. LS: Dr. San has received grants and served as consultant, advisor or CME speaker for the following entities: AstraZeneca, Bristol-Myers Squibb, Ferrer, Glaxo-Smith-Kline, Janssen, Lilly, Lundbeck, Otsuka, Pfizer, Roche, Sanofi-Aventis, Servier, Shire and the Spanish Ministry of Science and Innovation (CIBERSAM).

Consent for publication

Not applicable.

Ethics approval and consent to participate

The PLACID study will be conducted in compliance with independent ethics committee (IEC) and International Conference on Harmonisation (ICH) Good Clinical Practice Guidelines (GCP) and in accordance with all applicable European Community regulations regarding clinical safety data management. In addition, the study will adhere to all local regulatory requirements and requirements for data protection applicable for all participating centres. All trial centres will be required to provide a written and dated approval/favourable opinion from the relevant IEC. A list of all the ethics committees that have approved the study are listed in the supplementary document named Additional file $1 . x \mathrm{~s}$.

The Investigator will explain the benefits and risks of participation in the study to each patient and obtain written informed consent. Written informed consent will be obtained prior to the patient entering the study and before initiation of any study related procedure. The informed consent process will start with an assessment of the patient's capacity to provide informed consent. If the patient demonstrates adequate understanding and consent capacity per the Investigator's judgement, written informed consent will be obtained, with documentation of the patient's understanding of the key issues. Patients considered by the Investigator or other appropriately qualified site staff to have impaired consent capacity will not be included in the study.

\section{Publisher's Note}

Springer Nature remains neutral with regard to jurisdictional claims in published maps and institutional affiliations.

\section{Author details}

'Mental Health Department, Parc Sanitari Sant Joan de Deu, CIBERSAM, Carrer Camí Vell de la Colonia, 25 Sant Boi de Llobregat Barcelona, Spain. ${ }^{2}$ Ferrer, Barcelona, Spain. ${ }^{3}$ Hospital Clínic, Institute of Neuroscience, University of Barcelona, IDIBAPS, CIBERSAM, Barcelona, Spain. 
Received: 9 February 2017 Accepted: 29 March 2017

\section{Published online: 04 April 2017}

\section{References}

1. World Health Organization. Trastornos mentales. Available at: http://www. who.int/mediacentre/factsheets/fs396/es/.

2. Garriga M, Pacchiarotti I, Kasper S, Zeller SL, Allen MH, Vazquez G, et al. Assessment and management of agitation in psychiatry: expert consensus. World J Biol Psychiatry. 2016;17:86-128.

3. Hankin CS, Bronstone A, Koran LM. Agitation in the inpatient psychiatric setting: a review of clinical presentation, burden, and treatment. J Psychiatr Pract. 2011;17:170-85.

4. Nordstrom K, Zun L, Wilson M, Vs M, Ng AT, Bregman B, et al. Medical evaluation and triage of the agitated patient: consensus statement of the American Association for Emergency Psychiatry Project BETA medical evaluation workgroup. West J Emerg Med. 2012;13:3-10.

5. Citrome L, Volavka J. The psychopharmacology of violence: making sensible decisions. CNS Spectr. 2014;19(5):411-8.

6. Huber CG, Lambert M, Naber D, Schacht A, Hundemer HP, Wagner TT, et al. Validation of a clinical global Impression scale for aggression (CGI-A) in a sample of 558 psychiatric patients. Schizophr Res. 2008;100:342-8.

7. Jimenez X, Espila JL, Gallant J. Activation codes: past, present and future in Spain. Emergencies. 2011;23:311-8.

8. Allen MH, Currier GW. Use of restraints and pharmacotherapy in academic psychiatric emergency services. Gen Hosp Psychiatry. 2004;26:42-9.

9. Garrido Viñado E, Lizano-Díez I, Roset Arissó PN, et al. El coste económico de los procedimientos de contención mecánica de origen psiquiátrico en España. Psiquiatr Biológica. 2015;22:12-6.

10. Rubio-Valera M, Luciano JV, Ortiz JM, Slavador-Carulla L, Gracia A, Serrano-Blanco A. Health service use and costs associated with aggressiveness or agitation and containment in adult psychiatric care: a systematic review of the evidence. BMC Psychiatry. 2015;15:417.

11. Battaglia J. Pharmacological management of acute agitation. Drugs. 2005;65:1207-22.

12. Currier GW, Trenton A. Pharmacological treatment of psychotic agitation. CNS Drugs. 2002;16:219-28.

13. Currier GW, Allen MH, Bunney EB, Daniel DG, Francis A, Jagoda A, et al. Standard therapies for acute agitation. J Emerg Med. 2004;27(4 Suppl):S9-S12.

14. Abilify ${ }^{\circledR} 7.5 \mathrm{mg} / \mathrm{mL}$ solution for injection. Summary of Product Characteristics. Otsuka Pharmaceutical Europe Ltd. 4 June 2009. http://www. ema.europa.eu/docs/en_GB/document_library/EPAR_-_Product_ Information/human/000471/WC500020170.pdf.

15. Kwentus J, Riesenberg RA, Marandi M, Manning RA, Allen MH, Fishman RS, et al. Rapid acute treatment of agitation in patients with bipolar I disorder: a multicenter, randomized, placebo-controlled clinical trial with inhaled loxapine. Bipolar Disord. 2012;14:31-40.

16. Lesem MD, Tran-Johnson TK, Riesenberg RA, Feifel D, Allen MH, Fishman R, et al. Rapid acute treatment of agitation in individuals with schizophrenia: multicentre, randomised, placebo-controlled study of loxapine. Br J Psychiatry. 2011:198:51-8.

17. Popovic D, Nuss P, Vieta E. Revisiting loxapine: a systematic review. Ann General Psychiatry. 2015;14:15.

18. Dundar Y, Greenhalgh J, Richardson M, Dwan K. Pharmacological treatment of acute agitation associated with psychotic bipolar disorder: a systematic review and meta-analysis. Hum Psychopharmacol. 2016;31:268-85.

19. Vieta $\mathrm{E}, \mathrm{Cruz} N$. Head to head comparisons as an alternative to placebo-controlled trials. Eur Neuropsychopharmacol. 2012;22:800-3.

20. Zimbroff DL, Marcus RN, Manos G, et al. Management of acute agitation in patients with bipolar disorders: efficacy and safety of intramuscular aripiprazole. J Clin Psychopharmacol. 2007;27:171-6.

21. Adasuve ${ }^{\oplus} 9.1 \mathrm{mg}$ inhalation powder. Summary of Product Characteristics. Alexza UK Ltd. 20 February 2013.

22. Andrezina $\mathrm{R}$, Josiassen RC, Marcus RN, et al. Intramuscular aripiprazole for the treatment of acute agitation in patients with schizophrenia or schizoaffective disorder: a double-blind, placebo controlled comparison with intramuscular haloperidol. Psychopharmacology. 2006;188:281-92.

23. Guy W. Clinical global impressions (CGI) ECDEU assessment Manual for psychopharmacology, US Department of Health Education, and welfare publication (ADM); 1976. p. 76-338.

24. Atkinson MJ, Sinha A, Hass SL, Colman SS, Kumar RN, Brod M, et al. Validation of a general measure of treatment satisfaction, the treatment satisfaction Questionnaire for medication (TSQM), using a national panel study of chronic disease. Health Qual Life Outcomes. 2004;2:12.

25. Spyker DA, Munzar P, Cassella J. Pharmacokinetics of loxapine following inhalation of a thermally generated aerosol in healthy volunteers. J Clin Pharmacol. 2010;50:169-79.

26. Allen MH, Felfel D, Lesem MD, Zimbroff DL, Ross R, Munzar $P$, et al. Efficacy and safety of loxapine for inhalation in the treatment of agitation in patients with schizophrenia: a randomized, double-blind, placebo-controlled trial. J Clin Psychiatry. 2011;72:1313-21.

27. Busner J, Steven D, Targum MD. The clinical global impressions scale: applying a research tool in clinical practice. Psychiatry (Edgmont). 2007;4(7):28-37.

28. Montoya A, Valladares A, Lizan L, et al. Validation of the excited component of the positive and negative syndrome scale (PANSS-EC) in a naturalistic sample of 278 patients with acute psychosis and agitation in a psychiatric emergency room. Health Qual Life Outcomes. 2011;9:18.

\section{Submit your next manuscript to BioMed Central and we will help you at every step:}

- We accept pre-submission inquiries

- Our selector tool helps you to find the most relevant journal

- We provide round the clock customer support

- Convenient online submission

- Thorough peer review

- Inclusion in PubMed and all major indexing services

- Maximum visibility for your research

Submit your manuscript at www.biomedcentral.com/submit
) Biomed Central 